\title{
5 Artificial intelligence and the offense-defense balance in cyber security
}

\author{
Matteo E. Bonfanti
}

Cyber security is a quickly evolving domain which is constantly shaped by technological, policy, regulatory, economic, and social developments. Homomorphic encryption, quantum computing, and block chain represent some of the latest innovative approaches and applications, which promise to change the practice of cyber security. Nowadays, topping the list is "artificial intelligence" (AI), a variegated suite of concepts, methods, and tools whose transformative capacity is widely celebrated but has yet to be fully seen and understood.

Although researched and developed for some decades, AI has become significantly attractive for cyber security stakeholders only in recent times, when latest advancements in this technological field have shown the potential to impact on cyber security (Cussins Newman 2019: 14 ff.). Such growing attention on AI and its intersections with the security of cyberspace is reflected by the increasing number of dedicated initiatives both governmental and private organizations have promoted over the last few years.

However, the time needed for the outcomes of the AI-induced transformation on cyber security to become more tangible and widespread (the "when" question) is contested. While some are prudent about claiming revolutionary change, others are very enthusiastic. Consider, for example, the statement made by the (former) Commander of US Cyber Command and Director of the National Security Agency, Admiral Michael Rogers, a few years ago: “Artificial Intelligence and machine learning - I would argue - is foundational to the future of cyber security [...] It is not the if, it's only the when to me" (Allen and Chan 2017: 18). Admiral Rogers's thoughts have been seconded by many representatives of the cyber security community across the world (Osterman Research 2018).

As relevant as the "When" question are the "How" and the "To what extent" questions: How and to what extent will AI transform cyber security? How and to what extent will it enhance the protection of individuals, organizations, and their cyber-dependent assets from hostile threat actors? How and to what extent will it introduce novel vulnerabilities and enable additional typologies of actions? How and to what extent will it impact on cyber offense and defense? How and to what extent will cyber security stakeholders be able to deal with AI-induced changing risks and opportunities? This chapter will shed light on the difficulty to answer these questions, especially if one looks for concrete responses that are

DOI: $10.4324 / 9781003110224-6$ 
valid in the mid-/long term, but at the same time, it will take stock of the contemporary knowledge about when, how, and to what extent AI and cyber security are converging.

A first subsection defines artificial intelligence - then, the chapter introduces recent $\mathrm{AI}$ initiatives in the private sector and on the state level. A third subsection moves on to outline the uncertainties of the innovation process, the underlying difficulty to predict the future of AI in cyber security. However, some statements about the future of AI and cyber security are possible. In its subsequent sections, this chapter identifies several trends through the study of selected scientific and technical literature discussing the present - both embryonic and more mature - applications of AI-based solutions, which - from a technical point of view - promise to affect cyber security in the coming few years. The chapter acknowledges that the governance of AI innovation, in general, and with regard to cyber security, in particular, is still at its infancy and fragmented. The governance models that have been emerging so far will develop further under the pressure of the forces which are displayed by the actors mentioned earlier, and their mutual power relations. More initiatives are therefore to be expected; their effects will have to be assessed. At the moment, it seems that the impact of AI innovation on cyber security is still relatively more driven by the achieved, and yet to be achieved but possible, technological advancements than by emerging standards or regulations.

However, there are some early and promising applications of AI to cyber security which allow the making of an informed, although general, guess on what to expect in the near-term future. In particular, they allow to speculate on how the cyber security landscape might look like within the next 3-5 years. AI will enrich the cyber threat landscape - both in quantitative and qualitative terms. It will likely increase the number of cyber threat actors, offer them additional exploitable vulnerabilities and targets, as well as boost their malevolent actions. Conversely, AI will serve the defense from those threats by enabling the discovering of unknown vulnerabilities, the detection of malicious cyber activities, the implementation of countermeasures, and by augmenting the shortage of human professionals available to address imminent challenges.

Simply put, artificial intelligence will integrate and support cyber defensive and offensive activities, which may involve both the logical and the semantic layers of the cyberspace. Most of the features and functionalities which make artificial intelligence appropriate to cyber defense also make it suitable to offense. This is for example the case of the employment of AI to produce targeted cyber intelligence, which can be consumed for both protective and aggressive purposes. It is difficult to establish which application, defensive or offensive, will benefit relatively more from the integration of AI capabilities. It will depend on the capacity of single cyber security stakeholders (governmental or private) to master AI and leverage it for their intended purposes. It will also depend on their overall capacity to identify, understand, and address the risks, threats, and opportunities stemming from the integration of these technologies into cyber defensive or offensive systems. 


\section{Looking for a (working) definition of artificial intelligence}

There is currently no consensus on what AI exactly is, and often definitions come with controversy. One may claim there is actually no need for any clearcut, comprehensive, and crystallized definition, given that AI can be seen as a dynamic cluster of several technological concepts and approaches. Furthermore, progresses in this field will make any definition quickly obsolete. On top of that, focusing on notions and definitions might be less important than elaborating on the practical adoptions of AI, whatever this latter is exactly and regardless of any consensus about its core notion.

However, having at least a basic and shared understanding of artificial intelligence seems important because it can help relevant stakeholders to be consistent, transparent, and more effective when they promote programs, initiatives or take actions concerning AI at the policy, legal, operational, and other levels. For the purpose of the present chapter, such understanding is particularly functional to the analysis of the implications of AI research and applications for cyber security (and vice versa).

AI can be loosely defined as the ability, displayed by certain artificial/synthetic systems or "agents", to perform tasks that would require natural (human) intelligence (Coombs 2018; UNIDIR 2018; Russell and Norvig 2016) or, in particular, rationality (AI HLEG 2019). ${ }^{1}$ Broadly, it is a field of studies devoted to making artificial systems/agents able to accomplish missions which are commonly thought to require a certain degree of understanding and reasoning (Russell and Norvig 2016). ${ }^{2}$

There are different approaches to provide these agents with intelligence or make them rational, one of which is machine learning (ML). This approach is variously characterized as either a sub-field of AI or a separate field, and refers to the development of systems that improve their performance on a given task over time through experience and learning. ${ }^{3}$ The core components of machine learning solutions are learning algorithms, data, and powerful computational capabilities for training algorithms. ${ }^{4}$ An advanced approach to machine learning employs deep neural networks, i.e. numerous layers of algorithms (model) - each providing a different interpretation to the data they are fed on (The MITRE Corporation 2017). ${ }^{5}$ Such an approach is generally referred to as "deep learning".

It should be noted that to the extent to which an artificial system or agent learns on its own (independently from its designer's constant input) how to compensate for partial or incorrect prior knowledge, it is autonomous. As an attribute of intelligence/rationality, autonomy is the ability of an agent to determine and implement a course of action that is aimed to a certain goal, with no or less external guidance and oversight (Russell and Norvig 2010: 39). Regardless of the specific approach employed to make agents intelligent/rational and autonomous, all AI in existence today and that will be available in the near-term future fall under the broad category of Narrow Artificial Intelligence (UNIDR 2018). "Narrow" refers to the fact their intelligence/rationality is limited to a single task or domain of knowledge. Their autonomy is also reduced, meaning that human control is 
still prevalent. Different from nowadays available narrow AI are futuristic agents commonly labeled Artificial General Intelligence (AGI) (or "third wave AI", "transformative AI", "true AI"). "General" refers to the capacity to perform multiple tasks autonomously by employing a degree of intelligence/rationality equal if not superior to the one displayed by human beings. ${ }^{6}$

In the light of the above basic understanding and for the purpose of the present chapter, artificial intelligence can be defined as artificial systems/agents implementing machine and deep learning approaches to perform a given task. In particular, when here discussed with regard to cyber security and cyber-related applications, AI refers to technological solutions integrating machine/deep learning approaches and capabilities to:

process (more quickly and efficiently than humans as well as with limited human supervision) large-constant flows of information and derive insight (often hidden to humans) which can inform a course of action relevant for cyber related purposes (to protect or compromise hardware, software, data or users).

The provided definition is flawed; it does not have any further ambition than serving the discussion in this chapter. Nevertheless, it integrates the basic properties of AI which have been identified by the relevant literature so far.

\section{Recent AI initiatives}

At the forefront of AI innovation there are multinational technology firms and other private corporations. Driven by profit, they keep on investing significant amount of resources (human, technological, organizational, and financial) in the development and commercialization of artificial intelligence. In their capacity of expertise, tools and services providers as well as through political lobbying, they contribute significantly to shape the AI and cyber security ecosystems.

As for governments, they have explicitly sustained AI advancements through multiple policy mechanisms at least since 2016. They have invested in AI infrastructures, encouraged academic education and professional training, funded scientific research, incentivized public-private partnerships and collaborations, as well as promoted standards through procurement or other policies. In consultation with the private sector and the broad civil society, they have in some cases sponsored the adoption of guiding principles or basic norms (e.g. fundamental rights, data privacy) to sustain "responsible" or "trustworthy" innovation in this technological field (European Commission 2019). In many countries - e.g. China, the United Kingdom, Canada, India, Japan, France - governments orient their actions toward the acquisition of AI capabilities according to wide-scope national AI strategies, most of which address cyber security as one promising field of application (Cussins Newman 2019: $34 \mathrm{ff}$; OECD.AI). ${ }^{7}$ These strategies are then complemented by further policy instruments or other technical documentation tackling sectoral applications of AI. 
In general, governmental policies and their implementing actions pursue the threefold objective of encouraging the uptake of AI, maximizing its benefits, and minimizing the associated risks. As far as cyber security is concerned, policies aspire to make AI capabilities available to relevant national cyber security stakeholders (mainly public and private organizations) and ensure they can resort to these capabilities to gain an advantage over their competitors. An advantage which can make the difference in terms of power relations, i.e. in the capacity of such stakeholders to safeguard their assets and promote their interests in or through the cyberspace.

The abovementioned set of initiatives suggests that governments and private corporations largely believe in the transformative capacity of AI and are aware of the importance of mastering it in the coming years. Furthermore, they show there is general consensus on, and expectations for, the role AI will play in shaping future practices of cyber security (and security in general). Many independent experts and academics do also consider AI a sort of game changer for cyber security or, less emphatically, agree on the impact these technologies will have on this domain. The difficulty to tackle the "When?, "How"?, and "To what extent"? questions lies in the inherent uncertainties of the innovation process.

\section{Innovation and uncertainty}

Innovation keeps on developing quite fast under the pressure of several forces that are displayed by multiple actors (public/private researchers, developers and providers; policy and regulatory authorities at the domestic or supranational level; security/military agencies; the broad civil society). Making predictions on the mid-/long-term outcomes of such processes is hard. In some ways, it seems to neglect the rapid progress AI research and applications have undergone in the last couple of years only. ${ }^{8}$ Progress that will probably continue quite fast, boosted by growing public and commercial investments in the field (Fischer and Wenger 2019). In addition, AI is not the only technological component which promises to change cyber security. There are further technologies displaying a similar transformative capacity. AI will interact with these technologies in a way that can be hardly predicted from now. As a consequence, there are few chances to establish a priori the whole spectrum of possible interactions of AI with these technologies and foresee the overall impact on cyber security.

Furthermore, advances in AI should be understood as socio-technical phenomena that are more than the sum of technological capabilities and scientific/technical knowledge (Cussins Newman 2019: 6). Progress made in AI research and applications, and their implications for cyber security, are inevitably shaped by the models of governance which emerge from the formal/informal, fragmented/ coordinated, and often unbalanced interactions among public authorities, private organizations, and the civil society. Progresses will be also influenced and driven by the above actors' assessment of the risks and opportunities stemming from the deployment of AI for cyber or other security purposes.

To note, risks and opportunities are not to be understood in narrow technological terms only, e.g. as strictly pertaining to the functioning of AI tools, their safety 
and efficiency. They are broader and involve further aspects of the communities which are affected by the employment of AI. At the higher level, they involve nations' economic integrity and well-being, social cohesion, diplomatic relations or political stability. The governance of such risks and opportunities will therefore reflect individual and collective assessments, visions, values, interests, and challenges. In sum, given the trajectory of AI innovation remains still uncertain and determined by the interaction of multiple players and forces, it is hard to predict how it will impact on cyber security, especially in the mid-/long term.

\section{The security and cyber security relevance of artificial intelligence}

However, a series of elements make artificial intelligence research and application relevant from a broad security perspective (Cussins Newman 2019: 11ff; Brundage et al. 2018: 16-18; 24-29). These are more or less the same elements that raise implications for cyber security. Like many other technologies, AI is "dual-use" meaning that it can be employed for both civil and military purposes, and to do good or harm. It can for example integrate weapon, surveillance, warning, or other types of systems which find military application (defense or offense). It can also upgrade the tools used by civilian security agencies for contrasting threats to public safety and order. Actually, the realm of potential military-security applications and further spillovers are wide and diverse. In principle, such applications can leverage a set of properties/features that are intrinsic to AI technologies or, at least, they aspire to display.

Indeed, these technologies are designed to be both "efficient" and "scalable", as well as "adaptable" to the environment in which they can operate. Efficiency entails that, once deployed, AI can in principle complete a task more quickly or cheaply than humans. ${ }^{9}$ Scalability implies that, by increasing one of its underlying components (e.g. computing power), AI may become able to handle a growing amount of work, i.e. complete many more instances of a given task. AI technologies are also designed to be self-adaptable, meaning that they are prone to adjust their behavior/functioning according to changes in the environment they operate or the circumstances they are confronted with. The listed properties make AI appealing to different types of actors (state or non-state) because, among other things, they can be exploited for offensive, defensive, or other security-related goals.

Another element that makes AI relevant from a security standpoint concerns the fact that research and developments in this field lend themselves to rapid diffusion. Algorithms, datasets, processing capabilities - i.e. the basic components of AI - and, in general, relevant scientific findings are available to many research communities across the world. These communities are quite open in terms of knowledge transfer or capabilities sharing. Openness and availability support the so-called process of "democratization" or "commoditization" of artificial intelligence. This implies that there are many and geographically distributed stakeholders who can be empowered by advancements made in AI. Some of them might 
exploit these advancements for harmful purposes or, in general, to gain a competitive advantage in the security-military domain.

To the above listed elements, one should also add that AI comes with a number of novel, yet unresolved and often unknown vulnerabilities, which may have severe implications from a safety and security perspective. These vulnerabilities might be the cause of incidents or pave the way to both known and unknown malicious forms of exploitation (Patel et al. 2019). Experts are already aware of the risk AI-integrated systems can be subjected to, the so-called "data poisoning attacks" (introducing training data that causes a learning system to make mistakes) or "adversarial examples" (inputs designed to be misclassified by machine learning systems) (Gu et al. 2019). They are also aware there is a wide range of potential malicious exploitations that has still to be fully explored. ${ }^{10}$

What do the above elements - which make AI relevant for security - imply from a cyber-security point of view? To provide a simple answer, it is possible to frame the cyber security implications in terms of the possible threats and risks associated with the use/abuse of AI by cyber (threat) actors (Allen and Chan 2017). Of course, this is only one way to look at the issue. Another way would be to highlight the opportunities that AI may offer in terms of enhanced capacity to pursue cyber security-related goals as well as to cope with emerging threats. Indeed, depending on the adopted perspective, someone's security risks/threats are someone else's opportunities/advantages. Keeping this in mind but looking at the risk side, one may say that, absent the adoption of any substantial preventive measure, the availability and deployment of AI components could (i) expand existing cyber threats (quantity); (ii) alter the typical character of these threats (quality); (iii) introduce new and unknown threats (quantity and quality).

With regard to the first typology of implications, AI could expand the set of actors who are capable of carrying out malicious cyber activities, the rate at which these actors can carry the activities out, and the set of plausible targets/victims. This claim follows the efficiency, scalability, adaptability of AI technologies, as well as the "democratization" of research and development in this field. In particular, the diffusion of AI components among traditional cyber threat actors could increase the number of entities for whom become affordable carrying out particular attacks, especially those that are premised upon advanced social engineering, adversarial vulnerability detection, and spear-phishing. ${ }^{11}$ Given that AI applications are also scalable, actors who already possess the resources to carry out the above attacks may gain the ability to do so at a higher rate. It may become worthwhile for them to attack targets who otherwise would not make sense to attack from the standpoint of prioritization or cost-benefit assessment.

From a qualitative point of view, AI-enabled/powered cyberattacks could also feature in more effective, finely targeted, and sophisticated actions than those possible without using AI components. As per the increased effectiveness, it derives from the attributes of efficiency, scalability, and adaptability of these solutions. More finely targeted attacks could be the consequence of the efficient and scalable employment of AI to identifying and scrutinizing potential targets. 
Finally, artificial intelligence could enable a new variety of malicious activities and attacks, which exploit the vulnerabilities introduced by these technologies. In other words, the diffusion and integration of AI components in diverse types of cyber-related systems will introduce more hackable things into the virtual and physical world. In this latter regard, it is worth making an important observation. Large part of the public debate on artificial intelligence and cyber security concerns how AI research and applications do or will impact on cyber security, how they will affect the cyber threat landscape and increase risks and opportunities for cyber stakeholders (Loaiza et al. 2019). That is only one dimension of a much broader and articulated interaction between the two domains, however (IEEE and Syntegrity 2017).

Less discussed - except for experts' fora - is how cyber security itself is relevant to, and has a stake in, AI research and applications (I IEEE and Syntegrity 2017). Indeed, to the maximum possible extent, immunity from cyber threats should be an attribute of AI components. In order to preserve their proper functioning, reliability, and integrity, as well as avoid nefarious effects, AI tools should be safeguarded against cyber incidents or attacks. This implies that cyber security is a major and ongoing priority to the development and implementation of AI solutions (Spring et al. 2019: 11). Basically, it means that - when appropriate - relevant cyber security practices need to be applied. Reference is made to a set of actions and procedures aimed at promoting security (ideally "by design") from cyberattacks or incidents that leverage AI or other types of vulnerabilities (Brundage et al. 2018). For example, "red teaming" and "stress testing" should be carried out when AI solutions are at the research or development stage or piloted. Such testing aims at exploring what an actual cyberattack or induced incident might look like. It might help in discovering and fixing potential vulnerabilities.

Testing AI against cyberattacks might also be useful to better assess the skills and capabilities required to carry them out, to draw cyber threat scenarios, and check how defense should work in practice (US NSTC 2020). Another example of cyber security practice which can be beneficial to building secure/safe AI solutions consists in the responsible disclosure of systems' vulnerabilities, especially the so-called " 0 days". ${ }^{12}$ It consists in disclosing vulnerabilities to the affected parties before disseminating them widely. The goal is to provide these parties with the opportunity to remedy (patching). One could imagine the establishment of shared procedures for confidential reporting on vulnerabilities which are discovered in AI solutions (including potential adversarial inputs, and other types of exploits). Evidently, the adoption of the above or further cyber security practices, as well as the promotion of broad cyber hygiene programs with specific requirements for AI research, development, and application, represent a matter of governance.

To sum up, AI is relevant for cyber security (and vice versa). Depending on the adopted perspective, it may bring additional risks and threats, but also introduces further opportunities. To better understand the origins of such risks, threats, and opportunities, it is useful to look at the potential applications of AI to cyber security from a more practical point of view. This requires examining the notion of AI and framing it within the cyber security context. 


\section{Artificial intelligence and cyber security: An overview of defensive/offensive applications}

Cyber security is a domain welcoming the deployment of AI-powered solutions by governmental authorities, private organizations, and other non-state actors (criminal organizations, hackers, politically motivated, or other groups), both for offensive or defensive purposes. ${ }^{13}$ As mentioned earlier, most of the features and functionalities which make AI appropriate to cyber defense applications are the same that make it suitable to cyber offense (US NSTC 2020). In the near-term future, one should therefore expect organizations adopting and implementing AI-based cyber defense capabilities to safeguard their assets (networks, information, people) from adversaries who might leverage both AI- and non-AI tools for offensive purposes. Similarly, there will be actors employing AI-powered cyber offense capabilities to compromise targets who might engage in AI- or non-AIintegrated cyber defense.

From both the defense and offense sides, AI-based cyber capabilities may support activities involving the logical dimension of the cyber space (software) and/ or its semantic dimension (information and data processed therein). As per the former dimension, AI components are expected to be employed either to protecting from or executing computer network operations (CNA, CNE). With regard to the latter, AI will likely support defense from or execution of so-called cyber information and influence operations (Cordey 2019).

\section{AI-powered defense/offense within the logical layer of cyberspace}

One promising application of AI concerns the production of targeted cyber intelligence to be consumed for defense or offense purposes, i.e. to enhance or compromise networks, information and users' security. As pointed out by the literature, these technologies are amenable to integrate several functions of the cyber intelligence process, in particular the "collection", "processing", and "analysis" of information (Bonfanti 2018; Galyardt et al. 2019). In particular, they can boost information gathering and widen its scope to multiple sources and several end points. They may also enhance processing operations, i.e. filtering and (probably) the technical validation of collected data. The former concerns the selection of significant items of information; the latter consists in their corroboration with additional data provided by other sources. AI can also support analysis by finding hidden patterns and correlations in the collected and processed data.

By integrating AI capabilities into the listed functions, the cyber intelligence process will probably advance in terms of automation and speed. To avoid misunderstandings, automation will not concern the whole course of actions that can be executed on the basis of the produced (finished) cyber intelligence. To the present date and for the next few years, it seems that no AI solution will be enough efficient and reliable in undergoing "fully" automatic (totally unsupervised) followup activities (Ridley 2018; Wirkuttis and Klein 2017). From a defense point of view, it is questionable that AI will support fully unsupervised technical response 
or remediation (such as automatic patching). It is even more unlikely that it will be in charge of more advanced and articulated forms of unsupervised response consisting in active cyber defense, hacking back, or other forms of automatic retaliation. The latter type of responses would be not only technically inefficient (given the attribution problem) but also undesirable given the political, legal, tactical/ operational, or other consequences it may generate. Consequences that need to be carefully pre-assessed by human decision-makers (IEEE and Syntegrity 2017).

The eligibility of AI components to product cyber intelligence will translate into specific applications at the tactical/technical and - to a relatively lesser extent - operational level of cyber security. As per the latter, AI will be probably used for defensive purposes to retrieve and process data gathered from network security analysis programs and correlate them against all known structured and unstructured information available in articles, threat feeds, books, blog posts, and other sources that provide cyber intelligence (Coombs 2018: $35 \mathrm{ff}$.). With regard to tactical/technical defense, AI will increasingly support cyber threats detection, analysis and, to a limited extent, prevention (Wirkuttis and Klein 2017; Apruzzese et al. 2018). ${ }^{14}$ It will integrate and enhance tools for anomaly/intrusion (networkbased attacks), phishing, and spam (emails) detection, threat characterization (malicious code), and users behavioral modeling. Another emerging/promising target for tactical defensive application of $\mathrm{AI}$ is automated vulnerability testing (Loaiza et al. 2019).

In particular, following a trend which has already started, AI components will upgrade Intrusion Detection Systems (IDS) that are aimed to discover illicit activities within a computer or a network (Buczak and Guven 2016; Apruzzese et al. 2018) ${ }^{15}$ spam and phishing detection systems aimed at reducing the waste of time and potential hazard caused by unwanted emails; and, finally, malware detection and analysis tools. As per the latter, AI will probably improve the discovery of modern and emerging malwares, which can automatically generate novel variants to elude traditional rule-based identification approaches. It will help in attributing these variants to the correct malware family thanks to its capacity to recognize some hidden patterns which are invisible to traditional or human-based analysis. AI components will also integrate multifactor authentication or verification systems. In particular, they will be used to detect a pattern of behavior for a particular user in order to identify changes in those patterns. Although promising, the described applications for anomaly and threat detection/analysis are tainted with both false negatives (Zetter 2019) and positives (Xin et al. 2018). As per the former, pilot testing or early deployment show they are still, and keep on being, a main problem. Even a false positive rate of $0.1 \%$ could account for hundreds of false alarms which are unbearable for many organizations (Apruzzese et al. 2018).

AI applications will also be used for cyber offensive purposes i.e. to compromise a target organization/user, its networks, and the data therein processed. They will enable more numerous and sophisticated cyberattacks (Brundage et al. 2018). As in the case of defense, AI approaches/components may generate cyber intelligence to prepare and implement attacks. They may improve the selection and prioritization of targets for cyberattacks involving social engineering. Thank 
to AI, potential victims' online information can be harvested and processed to automatically generate custom malicious websites/emails/links (based on profiling) (Brundage et al. 2018). As AI develops further, convincing chat bots may elicit human trust by engaging people in longer dialogues, and perhaps eventually masquerade visually as another person in a video/audio chat (see also below).

AI components will also enhance adversarial vulnerability discovery and exploitation. They will prompt sophistication in malware designing and functioning, as well as support their obfuscation. AI-powered malware can be able to evade detection and creatively respond to changes in the target's behavior. They will function as an autonomous and adaptive implant - which learns from the host it runs on in order to remain undetected, search for and classify interesting content for exfiltration, search for and infect new targets, and discovers new pathways or methods for lateral movement. Already in 2018, IBM researchers have developed a malware of this type they dubbed "DeepLocker" (Stoecklin 2018). ${ }^{16}$ Finally, AI will also be deployed to spoof authentication or verification systems (e.g. those integrating biometric identifiers) (Patel et al. 2019).

\section{AI-powered defense/offense within the semantic layer of cyberspace}

From an offensive point of view, artificial intelligence will likely enhance the planning and running of cyber information and influence operations, which are aimed at criminal or other illegitimate goals. By supporting automation, AI will boost digital information/intelligence gathering as well as surveillance of targets/ victims' online behavior (Bonfanti 2019). It will add on the set of tools to be employed to inform and influence adversaries through and within the cyberspace (Patel et al. 2019: 22 ff.), especially by leveraging social media platforms. As per the latter, AI can improve bots and social bots management and allow the production of messages to be targeted at those most susceptible to them (similar to behavioral advertisement) (Brundage et al. 2018).

Following an on-going trend, AI-based solutions - especially those integrating deep learning approaches - will be employed to create manipulated digital content to be propagated within online or other media. Such content - known as "synthetic media" or "deepfakes" - consists of hyper-realistic video, audio, imagery or text which cannot be easily exposed as fake through manual or other conventional forensic techniques (Collins 2019). ${ }^{17}$ Once generated, synthetic media may be abused, i.e. employed to cause harm to individuals, organizations, and the broad civil society. Harmful employment is already abundant and documented by the media. Mostly, it consists in the deployment of AI-doctored videos (generally of pornographic nature) for targeted cyber bullying/stalking and defamation via/ on online media (Chesney and Citron 2018). Less frequent - but probably on the rise in the near-term future - is the weaponization of synthetic media for cyberenabled blackmailing, scamming, corporate sabotage (via market or other types of manipulative operations), political propaganda, and warfare (Ajder et al. 2019). ${ }^{18}$ In these cases, synthetic media will play as add-ons to "individual/organizationoriented" or "communities-oriented" information operations (Bonfanti 2020). ${ }^{19}$ 
On the one hand, AI will integrate and enable the above activities, and on the other, it will contribute to counter them. Indeed, from a defensive point of view, AI can support the detection of, and response to, cyber influence and information operations. It can be deployed to monitor the online environment (e.g. social media platforms), identify the early signs of malicious operations (e.g. increasing bots or social bots activities) as well as discover altered digital content (e.g. synthetic media) (Knight 2019; Collins 2019).

\section{Conclusions}

In light of what has been presented so far, AI will affect cyber security in the coming years. In the short term, it will probably do so along the lines drawn above. It will support both cyber defense and offense. It is difficult to establish which application will benefit more: It will probably depend on the ability of cyber security stakeholders (governmental or private) to sustain, master, and make progresses in artificial intelligence research, development, and applications, as well as leverage AI for achieving specific goals. From a broader perspective, it will also depend on their capacity to identify, understand, and address the risks, threats, and opportunities stemming from the deployment of these technologies for cyber defense or offense.

With regard to governmental cyber security stakeholders, addressing the above risks and opportunities would in principle require them to establish adequate/effective/consistent governance frameworks for AI and cyber security. To the extent possible, frameworks should also be multidimensional and participated/inclusive. It means they should cover a wide array of aspects, ranging from the technological to the policy, regulatory, economic, and diplomatic ones. They could for example integrate standards for validation and certification of AI tools for cyber security, which may include the implementation/adaptation of already existing security/ safety best practices to identify and cope with AI vulnerabilities. They could also consist in specific norms and institutions to shape the openness (democratization) of AI research and put additional limits to knowledge/capabilities transfer in this technological domain.

If possible, governance frameworks should be inclusive too. They should be established with the proactive and (possibly) balanced collaboration of relevant domestic or international actors, i.e. representatives and domain experts from different sectors (public, private, the civil society) and disciplines (engineering, computer and data science, human and social science, etc.); they should involve and assign responsibilities to these actors with regard to the factual implementation of the envisaged model of governance.

To a variable extent, the governance frameworks that are emerging nowadays seem - at least in the intentions of their promoters/contributors - aimed at being both multidimensional and inclusive. It has still to be seen whether they will be adequate, effective, and consistent enough to tackle the risks, threats, and opportunities the employment of AI for cyber security raises. Yet little evidence is available to inform such an assessment. As already noted, AI and cyber security governance is still at its infancy. More actions are to be expected; their outcome is uncertain. 


\section{Matteo E. Bonfanti}

\section{Notes}

1 The term "agent" has been used with several nuances in AI and related fields (Davis 1999). According to Russell and Norvig (2010: $34 \mathrm{ff}$.), "An agent is anything that can be viewed as perceiving its environment through sensors and acting upon that environment through actuators". The adjective "artificial" is here used to mark their difference with "human" agents.

2 As a field of study, AI dates back to the 1950s when researchers started creating machines able to accomplish simple and then increasingly difficult tasks in autonomous ways, i.e. with less or no human supervision and control. Since the 1950s AI research has constantly progressed and found applications in different fields. Nowadays, AI is a critical component of widely used devices as automatic speech recognition, machine translation, spam filters, and search engines. Additional promising applications include driverless vehicles, digital assistants for medical diagnosis and treatment, and robotics. One effect of this continuous progress in AI research and applications is that only the most cutting-edge machines are usually labeled "intelligent". In other words, the standard for machines being considered "intelligent" is constantly evolving.

3 Not all AI systems use machine learning. However, for many applications, machine learning can be a powerful method for achieving intelligent behavior.

4 Machine learning can rely on different approaches, the most common are supervised, unsupervised, and reinforced.

5 Neural networks are supposed to work in a fashion similar to the human brain.

6 According to some great futurist minds there will be a point in time they call the singularity, when an artificial agent becomes smarter than humans in nearly every field (Coombs 2018).

7 At the supranational level, intergovernmental organizations like the European Union (EU) or the Organisation for Economic Co-operation and Development (OECD) are also favoring states' cooperation on AI. As far as the EU is concerned, in 2018 it has adopted the European Strategy on AI to address the opportunities and challenges deriving from the development and deployment of AI in different areas.

8 From a technical point of view only, there have been unimaginable improvements in the AI core infrastructures and components, i.e. computing power, algorithms design, standard software frameworks for faster replication of experiments, and the availability of large datasets.

9 This is not to say it is always the best deployable solution; other convenient approaches might better suit for the designated purpose.

10 According to Brundage et al. (2018: 17), there is another feature which makes AI relevant from a security perspective. By supporting automation and less degree of human control and supervision, AI solutions can allow a greater degree of distance of their users from the task to be performed; distance also from the effects/consequences it may have (harm it might cause).

11 Spear-phishing is more effective than regular phishing, which does not involve tailoring messages to individuals, but it is relatively expensive and cannot be carried out en masse. Generic phishing attacks are more profitable than spear-phishing despite their very low success rates but merely by virtue of their scale. Thanks to AI, attackers could conduct more effective spear-phishing attacks with greater frequency and at a larger scale.

12 In the cyber security community, " 0 -days" are software vulnerabilities that have not been made publicly known (and thus defenders have zero days to prepare for an attack making use of them).

13 As argued by Allen and Chan (2017: 20), "There is no obvious, stable outcome in terms of state vs. non-state power or offense vs. defense cyber advantage. It will depend on the balance of research and development investments by all actors, the pace of technological process, and underlying limitations in economics and technology". 
14 At the tactical/technical level, cyber intelligence improves the effectiveness of blocking technologies, helps infrastructure groups prioritize their patching activities, and allows security operations center (SOC) analysts to quickly and accurately decide which alerts require action. Operational cyber intelligence can accelerate incident response by providing rich context (e.g. attackers' modus operandi, tactics, techniques, procedures, capabilities) around an initial indicator.

15 These systems were traditionally based on patterns of known attacks, but modern deployments include approaches for anomaly and threat detection based on machine learning. These approaches can be used for the detection of botnets and of Domain Generation Algorithms (DGA). As per these latter, they are algorithms which generate domain names automatically, and are often used by an infected machine to communicate with external server(s) by periodically generating new hostnames. They represent a real threat to organizations because they allow to evade defenses based on static blacklists of domain names.

16 This AI-powered malware conceals its intent until it reaches a specific victim. It carries out its malicious action as soon as the AI component identifies the target through indicators like facial recognition, geolocation, and voice recognition. It is virtually impossible to exhaustively enumerate all possible trigger conditions for the AI model. What is unique about DeepLocker is that it uses AI (deep neural network) to unlock the attack. The malicious payload will only be unlocked if the intended target is reached. The AI model is trained to behave normally unless it is presented with a specific input: The trigger conditions identifying specific victims.

17 "Deepfakes" is a neologism (resulting from the merge of "deep learning" and "fake"). They can be generated with a variety of machine/deep learning techniques and approaches. Currently, the most popular is the Generative Adversarial Network (GAN).

18 In 2018-2019, there were already some cases of cybercriminals using AI-generated audio to impersonate a CEO's voice and convince subordinates into transferring funds to a scammer's account (the so-called "CEO-scam").

19 Both types of operations are intertwined and may be executed in combination; they can overlap. The former type of operations points to affect institutional or formal decision-making processes within selected organizations through deception and/or extortion/coercion. The latter target the society as a whole or specific social groups, in particular those groups' ideas, opinions, motivations, and beliefs, and aim at mobilizing them.

\section{References}

All links checked on August 20, 2021.

AI HLEG (High-Level Expert Group on Artificial Intelligence) (2019). A Definition of AI: Main Capabilities and Scientific Disciplines. Retrieved from: https://ec.europa.eu/ digital-single-market/en/news/ethics-guidelines-trustworthy-ai.

Ajder, H., Cavalli, F., Patrini, G. and Cullen, L. (2019). The State of Deepfakes: Landscape, Threats, and Impact. Retrieved from: https://regmedia.co.uk/2019/10/08/deepfake report.pdf.

Allen, G. and Chan, T. (2017). Artificial Intelligence and National Security. Cambridge, MA: Belfer Center for Science and International Affairs. Retrieved from: https://www .belfercenter.org/sites/default/files/files/publication/AI\%20NatSec\%20-\%20final.pdf.

Apruzzese, G., Ferretti, L., Marchetti, M., Colajanni, M. and Guido, A. (2018). On the Effectiveness of Machine and Deep Learning for Cyber Security. In: T. Minárik, R. Jakschis, and L. Lindström (eds). 10th International Conference on Cyber Conflict CyCon: Maximising Effects. Tallinn: NATO CCD COE Publications, pp. 371-390. 